\title{
Dendritic flux avalanches in a superconducting $\mathrm{MgB}_{2}$ tape
}

\author{
T Qureishy ${ }^{1}$, C Laliena ${ }^{2}$, E Martínez $^{2}$, A J Qviller ${ }^{3}$, J I \\ Vestgården $^{1}{ }^{4}, \mathbf{T} \mathbf{H}$ Johansen $^{1}{ }^{5}, \mathbf{R}$ Navarro $^{2}$ and $\mathbf{P}$ Mikheenko ${ }^{1}$ \\ ${ }^{1}$ Department of Physics, University of Oslo, P. O. Box 1048 Blindern, 0316 Oslo, \\ Norway \\ 2 Instituto de Ciencia de Materiales de Aragón, (CSIC - Universidad de Zaragoza), \\ C/ María de Luna 3, 5018 Zaragoza, Spain \\ 3 nSolution AS, Maries gate 6, 0368 Oslo, Norway \\ ${ }^{4}$ Norwegian Defence Research Establishment (FFI), Kjeller, Norway \\ ${ }^{5}$ Institute for Superconducting and Electronic Materials, University of Wollongong, \\ Northfields Avenue, Wollongong, NSW 2522, Australia \\ E-mail: thomashq@fys.uio.no
}

\begin{abstract}
Tapes of $\mathrm{MgB}_{2}$ with high critical current have a significant technological potential, but can experience operational breakdown due to thermomagnetic instabilities. Such events are routinely registered by magnetisation measurements, but were never observed by direct imaging techniques. Here we report magneto-optical imaging visualisation of a tape with reduced thickness and enhanced critical current density. The spatial structure of the thermomagnetic avalanche events was resolved, and the reproducibility and nucleation thresholds were determined. Avalanches appearing at low fields are small intrusions at the tape edges, while larger fields create more extensive avalanches having dendritic structures similar to those observed in thinfilm superconductors. In contrast to dendritic avalanches in thin films, the avalanches in the tapes show reproducibility to a larger extent.
\end{abstract}

Keywords: superconductivity, $\mathrm{MgB}_{2}$ tapes, magneto-optical imaging, dendritic avalanches

This is an author-created, un-copyedited version of an article published in Superconducting Science and Techonology.

IOP Publishing Ltd is not responsible for any errors or omissions in this version of the manuscript or any version derived from it.

The Version of Record is available online at DOI: 10.1088/1361-6668/aa9244

Supercond. Sci. Technol. 30 (2017) 125005 


\section{Introduction}

Soon after the discovery of superconductivity below $39 \mathrm{~K}$ in magnesium diboride $\left(\mathrm{MgB}_{2}\right)$ [1], the huge interest of the scientific community led to an extensive physical characterization of this type-II superconductor $[2,3]$. Improved magnetic flux pinning and hence a higher critical current density, $J_{\mathrm{c}}$, was achieved with carbon substitution for boron by chemical doping [4].

The powder-in-tube technology, extensively used in the fabrication of the firstgeneration high-temperature superconductors, was found to be useful for producing $\mathrm{MgB}_{2}$ tapes with high $J_{\mathrm{c}}$ and significant technological potential [5]. Iron is often used as metallic sheath material for $\mathrm{MgB}_{2}$ wires and tapes because of its good mechanical properties and chemical compatibility with $\mathrm{MgB}_{2}[6,7]$.

Magnetic flux jumps are observed in most type-II superconductors when applying a magnetic field at low temperatures. These abrupt events are caused by thermomagnetic instabilities [8, 9], which are triggered by Abrikosov vortices moving in the superconductor as an applied magnetic field or current is changed. In the motion, vortices dissipate heat, and if this heat is not removed quickly, more vortices depin and move into the sample dissipating even more heat. This self-amplifying process results in a thermomagnetic avalanche. Such events lead to jumps in magnetic moment of bulk $\mathrm{MgB}_{2}$ [10], as well as in $\mathrm{MgB}_{2}$ films [11], wires [12] and tapes [13].

The spatial structure of flux avalanches can be observed by magneto-optical imaging (MOI). Most commonly they have a dendritic structure, as found in thin films made of $\mathrm{Nb}[14], \mathrm{NbN}[15], \mathrm{MgB}_{2}[11,16]$ and $\mathrm{YBCO}[17,18]$, and also in foils of $\mathrm{Nb}[19]$. The detailed structure of dendritic avalanches is unpredictable. Yet, they have upper and lower thresholds for both increasing and decreasing magnetic fields [20]. In films of $\mathrm{MgB}_{2}$ cooled in zero magnetic field, dendritic avalanches have a threshold temperature of $10 \mathrm{~K}$ when applying an increasing magnetic field [16].

By combining MOI with magnetic moment measurements, we have investigated flux jumps in an iron clad carbon-doped $\mathrm{MgB}_{2}$ tape. We report observations of dendritic avalanches in the sample. Their spatial structures, reproducibility, threshold magnetic fields and temperature dependence are discussed. Threshold fields and their dependence on temperature are analysed and compared with theoretical models.

\section{Methods}

Four undoped and two carbon-doped $\mathrm{MgB}_{2}$ tapes were synthesized and characterized by MOI and magnetic moment measurements. Dendritic avalanches were found in only one of them, and the present paper focuses on detailed results for this particular sample. MOI results from the four undoped tapes are presented elsewhere [21]. Also an $\mathrm{MgB}_{2}$ wire was synthesized and characterized by magnetic moment measurements.

The $\mathrm{MgB}_{2}$ tape showing dendritic behaviour was manufactured by the powder-intube method using in situ reaction of ball-milled precursor powders, similarly to the 
process described in [21], but with oleic acid added to the powders before milling to improve its critical current density. Powders of $\mathrm{Mg}$ and $\mathrm{B}$ were mixed in stoichiometric ratio (1:2) with oleic acid in a Retsch MM 200 vibratory ball mill for 30 minutes. The amount of oleic acid was 10 wt. $\%$ of the $\mathrm{Mg}-\mathrm{B}$ mixture. In order to obtain optimum superconducting properties, the precursor powder was subsequently heat treated at $400{ }^{\circ} \mathrm{C}$ in an argon atmosphere for an hour [22]. After that, the powder was milled in a Retsch PM 100 planetary ball mill with balls of tungsten carbide, rotated at a speed of 200 revolutions per minute (rmp) for 1.5 hours. Every three minutes the milling was paused for one minute, and the rotation direction was changed.

Another carbon-doped $\mathrm{MgB}_{2}$ tape was prepared in a similar way, but instead of adding oleic acid to an Mg-B mixture, oleic acid was added to boron powder only. This powder and oleic acid were mixed in a Retsch MM 200 vibratory ball mill for 30 minutes. Then the mixture was heated at $400{ }^{\circ} \mathrm{C}$ in an argon atmosphere for an hour. After that, $\mathrm{Mg}$ powder was added and mixed in the same ball mill for 30 minutes. Finally, the mixture was milled in the Retsch PM 100 planetary ball mill under the same conditions as the other doped sample.

Four undoped $\mathrm{MgB}_{2}$ tapes were prepared as well, as described in [21]. The powders for the tapes were mixed in the Retsch PM 100 planetary ball mill with different rotation speeds $\omega$ and ball milling times $t$. The following values of these parameters: $t=0 \mathrm{~h}$ and $\omega=0 \mathrm{rpm}, t=1.5 \mathrm{~h}$ and $\omega=200 \mathrm{rpm}, t=3.0 \mathrm{~h}$ and $\omega=400 \mathrm{rpm}$, and $t=16.5 \mathrm{~h}$ and $\omega=400 \mathrm{rpm}$ were used.

The resulting powders were inserted into iron tubes with an inner and outer diameter of 4.5 and $5 \mathrm{~mm}$, respectively. The tubes were then sealed at both ends. After that, they were drawn through the formers in consecutive steps reducing the diameter down to $1.1 \mathrm{~mm}$ and finally cold-rolled into $2-\mathrm{mm}$ wide and $0.4-\mathrm{mm}$ thick tapes. During mechanical deformation, intermediate annealing at $550{ }^{\circ} \mathrm{C}$ for an hour in argon atmosphere was performed to reduce the iron sheath's work-hardening. Finally, the tape was also sealed at both ends and annealed at $670{ }^{\circ} \mathrm{C}$ for five hours in vacuum to form the final product.

After annealing, the tapes were cut into several pieces for characterization with different techniques. For MOI analysis, which is the main focus in the present work, 10 mm-long pieces were used. For measurements and structural analysis, one side of the tapes were polished to expose the superconducting core. The tape, analysis of which we present in this paper, was $\approx 50 \mu \mathrm{m}$ thick, and the other five tapes were $150-250 \mu \mathrm{m}$ thick. For comparison, pieces of the same $\mathrm{MgB}_{2}$ wires, without the rolling process, were also analysed. They were annealed with the same conditions as the tapes.

For characterization, the tape was mounted in a helium flow cryostat with a magneto-optical indicator film placed on top of it. The indicator is a Faraday-active layer, which in an optical system with crossed polarizers allows one to visualize magnetic flux that penetrates the specimen [23]. In our case it is a bismuth-substituted ferrite garnet film deposited on a gadolinium gallium garnet substrate with an aluminium mirror to reflect the light coming from the polarizer $[24,25]$. The superconductor with 
the indicator on top was cooled in zero magnetic field to a temperature below its critical temperature, $T_{\mathrm{c}}$. Then a field was applied perpendicular to its surface and increased to $\mu_{0} H=85 \mathrm{mT}$, while taking images every $0.85 \mathrm{mT}$. After that, the sample was heated above $T_{\mathrm{c}}$, and the process was repeated.

4-5 $\mathrm{mm}$ long samples of the same $\mathrm{MgB}_{2}$ wire and tape were characterized by vibrating sample magnetometers (VSM, Quantum Design PPMS-9T for the tapes and PPMS-14T for the wires) and SQUID magnetometer (Quantum Design 5T for both) when high magnetic fields were not necessary. After removing the iron sheath by mechanical polishing, the diameter and thickness of the wire and tape were $0.9 \mathrm{~mm}$ and $0.29 \mathrm{~mm}$, respectively. The isothermal magnetic hysteresis loops at $T=5 \mathrm{~K}$ in high fields were measured using PPMS systems. For the tape, a magnetic field was applied perpendicular to its flat surface and increased from $\mu_{0} H=0$ to 9 T. After that, it was decreased back to zero, and finally increased in the opposite direction to - $9 \mathrm{~T}$. In the case of the wire, the field was applied perpendicular to its axis and increased from 0 to $14 \mathrm{~T}$, decreased back to 0 and then increased in the opposite direction to - $14 \mathrm{~T}$. In order to analyse the virgin magnetic moment in more detail, additional experiments, for a wire and a tape, were carried out using the SQUID magnetometer. This allowed to analyse the initial magnetisation branch $m_{\text {ini }}\left(\mu_{0} H\right)$ in fields from 0 to $0.5 \mathrm{~T}$ for the tape and from 0 to $0.8 \mathrm{~T}$ for the wire, with steps in $\mu_{0} H$ of approximately $2 \mathrm{mT}$.

\section{Results and discussion}

In figure 1(a), magnetic moment $m$ of the tape is plotted as a function of applied magnetic field $\mu_{0} H$ at $T=5 \mathrm{~K} . m$ is smooth at high fields and contains flux jumps at low fields. Flux jumps are seen in the virgin curve and also in the reverse branches, after having applied the maximum field, in fields between +2.5 and $-2.5 \mathrm{~T}$. There is an additional ferromagnetic signal in the $m(H)$ curves caused by the presence of remaining sheath material, since it was difficult to remove it without damaging the sample. The inset in the lower-left corner shows several of the first flux jumps occurring in the virgin curve, including the first one at $\mu_{0} H=0.145 \mathrm{~T}$, marked by an arrow. The first flux jump is also shown in the inset in the lower-right corner.

Figure $1(\mathrm{~b})$ shows $m$ as a function of $\mu_{0} H$ at $5 \mathrm{~K}$ for the $\mathrm{MgB}_{2}$ wire. There are many flux jumps in the reverse branch in approximately the same field interval as in the tape, and also flux jumps up to about $3 \mathrm{~T}$ in the virgin curve of the wire. The inset to the left shows several of the first flux jumps, and an arrow points at the first one at $\mu_{0} H=0.185 \mathrm{~T}$. The inset to the right shows the first flux jump.

The exact flux jump pattern in $m(H)$ measurements depends on the ramp rate $\mu_{0} \mathrm{~d} H / \mathrm{d} t$, which is $13-30 \mathrm{mT} / \mathrm{s}$ for these measurements, depending on the system (note that the smaller the upper magnetic field of the magnet, the higher the ramp rate). Since both the wire and tape contain similar flux jump behaviour, it is likely that rolling the wire into the tape before the final annealing and polishing off the iron sheath is not the only cause of flux jumps in the tape. 

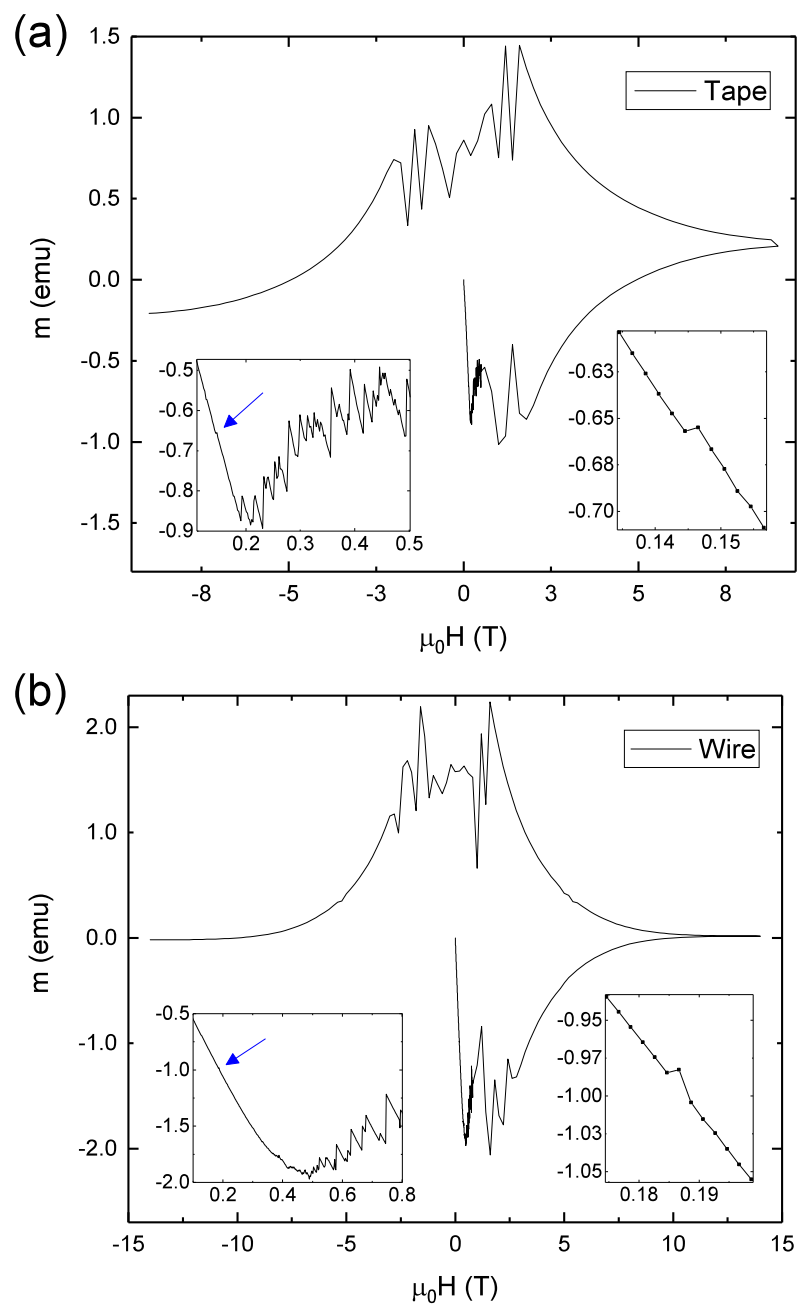

Figure 1. Magnetic moment as a function of applied magnetic field for (a) tape and (b) wire at a temperature of $5 \mathrm{~K}$. The insets in the lower-left corner of both figures show several of the first flux jumps in the virgin branch. An arrow points at the position of the first flux jump, which is shown in the inset in the lower-right corner of both figures. The units on the axes in the insets are the same as those in the main figures.

Since magnetic moment measurements give the average magnetic properties over the whole volume of the samples, it is desirable to visualize local magnetic fields by MOI to clarify the sample behaviour, even if the operating range of the indicator films is limited to low magnetic fields, in our case $85 \mathrm{mT}$, below the first detected jump in the magnetic moment measurements.

Figure 2 shows colour-coded MOI images of a section of the tape covering two thirds of its whole length. Such colour coding is a useful tool for demonstrating irreproducibility of flux penetration in MOI images, and it was previously used in [26]. The images were obtained at $3.7 \mathrm{~K}$ and different magnetic fields. Each image consists of a superposition of three images obtained under the same conditions (zero-field cooling to $3.7 \mathrm{~K}$ and applying the same magnetic field). They are presented in three different colours: red, green and blue (RGB). Grey colours in these images appear as a sum of 
these three colours. Brightness and contrast were optimized for each image individually. The images from the top to bottom correspond to increasing applied fields, namely to $\mu_{0} H=18.7,42.5,62.1$ and $84.2 \mathrm{mT}$, respectively. The inner region is dark, indicating zero flux, and the area outside of its borders is brighter, which shows that the tape expels magnetic field. The bright horizontal lines at the upper and lower edges of the tape are from the remnants of ferromagnetic sheath. Bright flux front propagating into the sample shows the advancement of magnetic flux.

Magnetic flux penetrates gradually into the sample from the edges and forms a critical state-like region with a non-smooth flux front. The flux front is also nonhomogeneous, especially in the upper-left corner, because of enhanced surface roughness in the tape there. In addition to gradual flux penetration, figure 2 clearly shows specific dendritic formations. These formations appear suddenly and have branch-like structures resembling lightning. The first dendrites are small, with very few branches. As the applied magnetic field increases, new and larger dendrites appear. At the highest applied fields, new ones have even more branches. Most of the dendrites have a non-reproducible appearance, as can be seen in the RGB images, where colours are not mixed. The parts of dendrites that are yellow, cyan or magenta were created by dendrites following the same path in two out of three measurements. This makes the pattern or parts of the pattern reproducible to some extent, although branches rarely follow the same path at the same applied fields. There are more dendritic avalanches on the left-hand side of the tape compared to the middle, especially at intermediate fields, because the surface is rougher and the tape is slightly thinner on the left side.

More information can be extracted from differential MOI images presented in figure 3. In this figure, the temperature and fields are the same as in figure 2 , with the only difference that preceding images are subtracted from them, which were obtained at slightly lower (by $0.85 \mathrm{mT}$ ) magnetic field. White dashed lines in the figure show the outline of the tape and were added as a guide to the eye. The insets in figure 3(a) show magnified images of two areas with enhanced brightness. As one can see, there are quite small dendritic avalanches at low applied magnetic fields, and several larger ones that appear at higher fields. The small dendrites at lower fields do not have as many branches as those appearing at higher fields.

Figure 4 shows images obtained at zero-field cooling to different temperatures of 4.0, 6.0, 8.0 and $10.0 \mathrm{~K}$ (from top to bottom), and applying magnetic field of $85 \mathrm{mT}$. Dendritic avalanches are seen in figures 4(a)-(c), but not in figure 4(d). The higher the temperature, the fewer dendritic avalanches are observed. The magenta colour of the dendrite in figure 4(c), which is a mixture of red and blue, shows that this particular dendrite is almost reproducible in two experiments. On the left end of the tape in figures $4(\mathrm{c})$ and $4(\mathrm{~d})$, there is a grey dendrite-like formation. However, magnetic flux penetrates gradually there while increasing the field, which indicates that it is not a thermomagnetic instability, but merely flux penetration into a crack in the sample. This colourless feature has a structure similar to the large dendritic avalanches, which indicates that cracks may play an important role in the resulting pattern of dendritic 


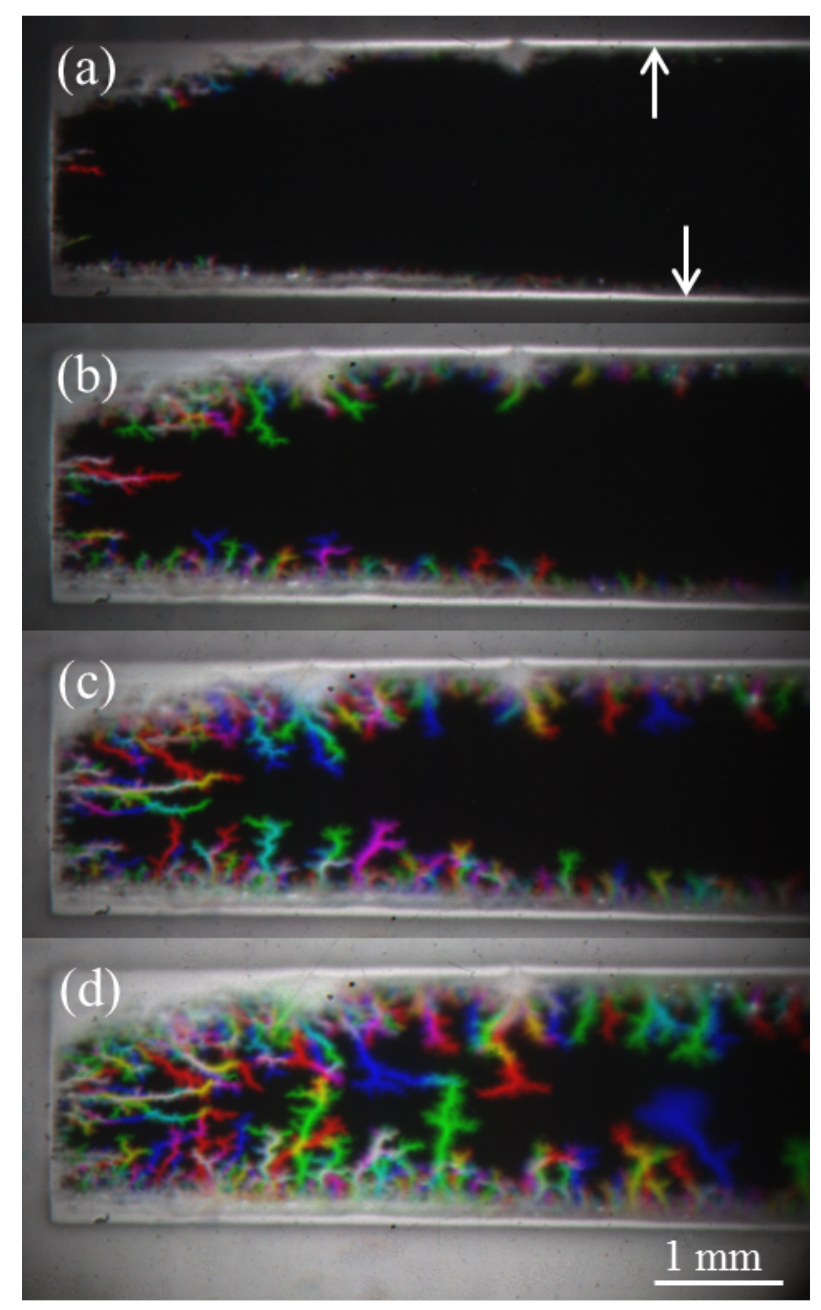

Figure 2. Colour coded MOI images obtained after zero-field cooling of $\mathrm{MgB}_{2}$ tape to $3.7 \mathrm{~K}$ and application of increasing perpendicular magnetic field of (a) 18.7, (b) 42.5 , (c) 62.1 and (d) $84.2 \mathrm{mT}$. The procedure was carried out three times, and each image is a superposition of three images obtained at the same temperature and applied magnetic field, plotted in red, green and blue. Grey colours appear as a sum of red, green and blue. In general, dendritic avalanches are irreproducible. White arrows point at the bright horizontal lines caused by remnants of ferromagnetic sheath.

avalanches. This is further supported by the similarity between the red dendrite in figure 3(b) and the colourless dendrite in figures 4(c) and 4(d). The red avalanche may have propagated into the crack. This could explain the few cases of reproducibility of dendritic formations in the sample.

The fields at which the first dendritic avalanche appeared at a given temperature were not the same in the three consecutive experiments. The lower threshold field $\mu_{0} H_{\text {thr }}$ for the appearance of dendritic avalanches as a function of temperature $T$ is shown in figure 5 . The temperatures are $3.7 \mathrm{~K}$ and every $0.5 \mathrm{~K}$ from 4.0 to $10.0 \mathrm{~K}$. At a given temperature, the red square, green triangle and blue circle correspond to the fields at which the first dendritic avalanche appeared in three different MOI experiments. 


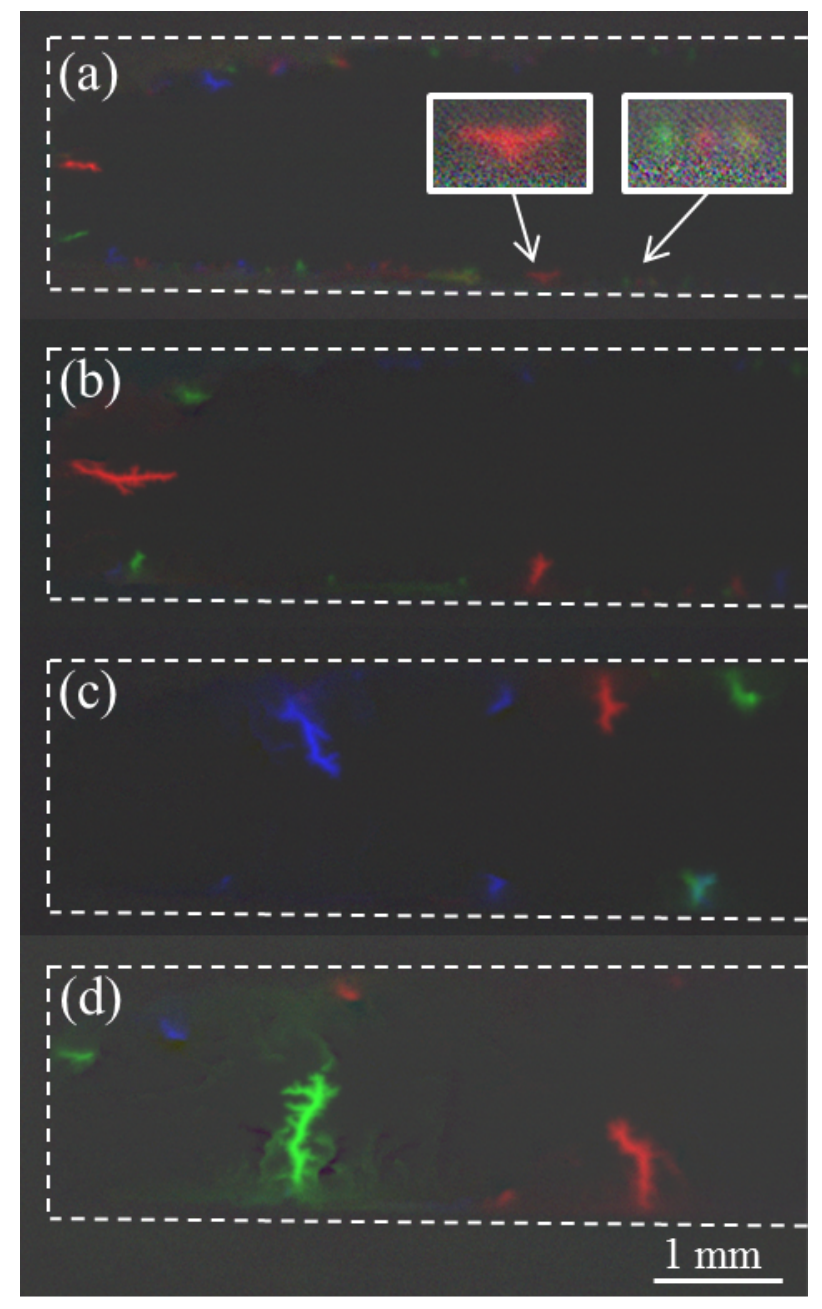

Figure 3. Differential RGB MOI images obtained after zero-field cooling the tape to $3.7 \mathrm{~K}$ and applying increasing perpendicular magnetic field. White dashed lines show the outline of the tape. The applied fields are the same as those in figure 2, namely (a) 18.7, (b) 42.5, (c) 62.1 and (d) $84.2 \mathrm{mT}$, and the images are obtained by subtracting preceding images taken at slightly lower (by $0.85 \mathrm{mT}$ ) magnetic field. The two insets in (a) show magnified images with enhanced brightness of several avalanches appearing from the bottom edge of the sample.

Dendritic avalanches were observed in all three experiments at temperatures up to $8.5 \mathrm{~K}$. At $9.0 \mathrm{~K}$ a dendritic avalanche was only observed in two of the experiments, and none were observed at 9.5 or $10.0 \mathrm{~K}$. Since the applied magnetic field, limited by $85 \mathrm{mT}$, was far below the field needed for full penetration at the temperatures of the experiments, we cannot determine exact threshold temperature $T_{\text {thr }}$ for the disappearance of dendritic avalanches, but can state that it is above $9.0 \mathrm{~K}$. In figure $5, \mu_{0} H_{\text {thr }}$ is merely constant at low temperatures, but increases a little with increasing $T$ up to $8.0 \mathrm{~K}$, and increases rapidly above $8.0 \mathrm{~K}$. This relationship between $\mu_{0} H_{\text {thr }}$ and $T$ is similar to what has been previously reported for 300-nm thick films of $\mathrm{MgB}_{2}$ [27] and 500-nm thick Nb films [28], but the rapid increase occurs at different temperatures in those experiments. The 


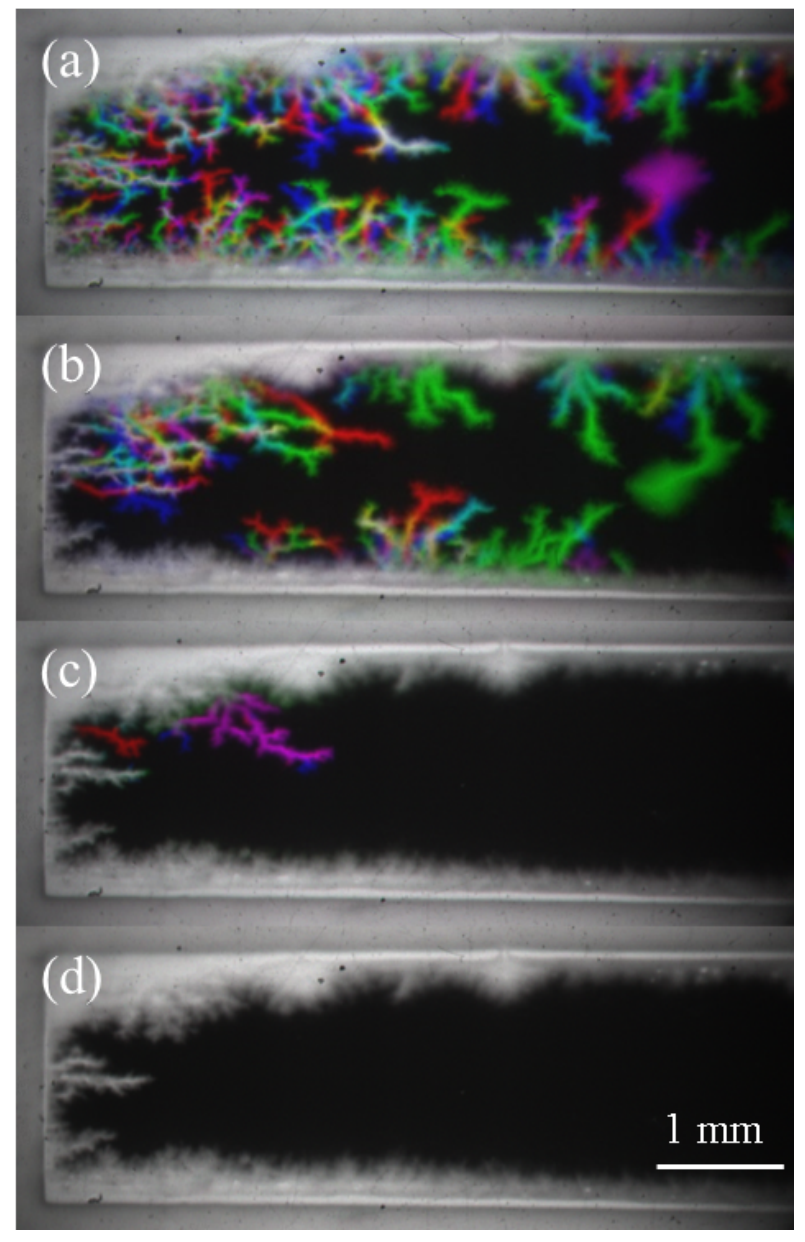

Figure 4. RGB MOI images after zero-field cooling of the tape to (a) 4.0, (b) 6.0, (c) 8.0 and (d) $10.0 \mathrm{~K}$ and subsequently applying perpendicular magnetic field of $85 \mathrm{mT}$, three times at each temperature.

variance of $\mu_{0} H_{\text {thr }}$ is also merely constant up to $T=8.0 \mathrm{~K}$ and then increases, which can be seen for 8.5 and $9.0 \mathrm{~K}$ in comparison with that at lower temperatures. Theoretical models $[27,29]$ predict that the threshold field should roughly scale with the critical sheet current, under otherwise identical conditions. Yet, our tape has a critical sheet current roughly five times larger than the thin film of [27] whereas the threshold field is similar. This indicates that conditions are not identical for the tape and the thin film and in particular we suspect that edge properties play an important role, because the tape edge is rough, and edge defects are known to lower the instability threshold [30, 31]. In our tape, the critical sheet current can be estimated from the flux penetration of the lower edge in figure 4 to be roughly $2.2 \cdot 10^{5} \mathrm{~A} / \mathrm{m}$.

It is also worth pointing out that the other five tapes, which did not show dendritic avalanches in MOI, displayed flux jumps registered by magnetometry at high fields. However, this field range is above the saturation field of the ferrite garnet films used in the present work [24, 25]. Note here that the tape in which dendritic avalanches was observed, is much thinner than the other five tapes. Thus, the threshold magnetic field 


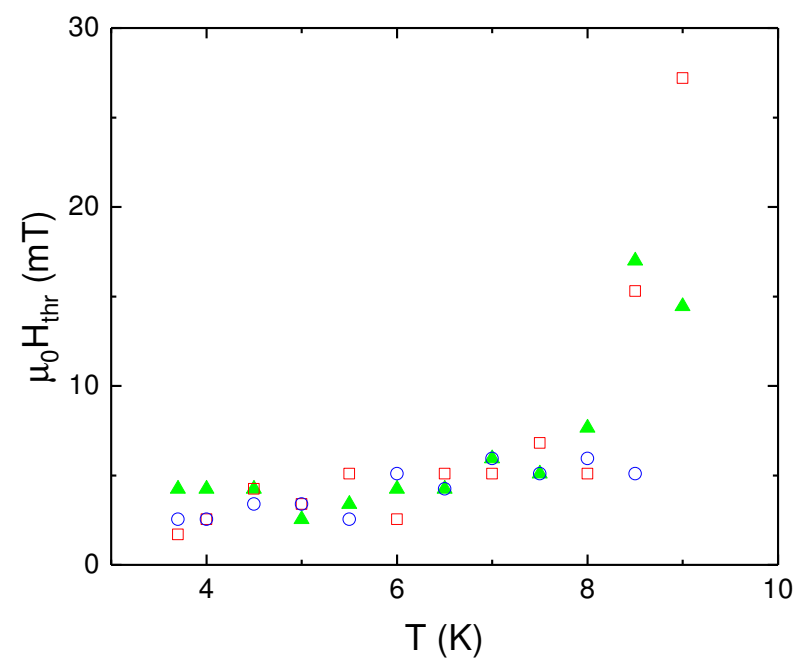

Figure 5. The lower threshold field for the appearance of dendritic avalanches as a function of temperature. At a given temperature, the red square, green triangle and blue circle correspond to the field of appearance of the first dendritic avalanche in three separate experiments.

did in this case fall within the operational range of the MOI technique.

Note finally that the threshold fields found from MOI experiments presented in figure 5 are much lower than $145 \mathrm{mT}$ found from the $m(H)$ measurements at $5 \mathrm{~K}$ in figure 1(a), because one could not see the smallest avalanches in magnetic moment measurements. If the magnitude of magnetic moment in $m(H)$ plots is lower than expected in areas where no flux jumps are seen, it is possible that there are in fact several flux jumps too small to be detected in such measurements, and being interpreted by the instrument as noise. MOI, although limited in operating range to low magnetic fields, can be employed to confirm the existence of such avalanches and visualize their propagation into the sample.

\section{Conclusions}

Flux jumps caused by thermomagnetic instability have been investigated in an $\mathrm{MgB}_{2}$ tape by MOI and magnetic moment measurements. Dendritic avalanches were observed with MOI and occurred at much lower magnetic fields than flux jumps seen in measurements of magnetic moment. The smallest thermomagnetic instabilities seen in MOI could not be observed in our magnetic moment measurements. The dendritic avalanches in the tape have similar properties to those appearing in thin films, but have relatively few branches and their patterns are in some cases partially reproducible. Most of them, however, are non-reproducible. The average size of new dendritic avalanches, as well as their branching, increases with increase of applied magnetic field. Upon increasing the temperature, the number of avalanches decreases. The lower threshold magnetic field for their appearance first increases slowly with increasing temperature and then increases rapidly above $8 \mathrm{~K}$. The lower threshold field is much lower than what 
is expected from theoretical models, indicating that the onset is dictated by the edge properties of the tape.

\section{Acknowledgments}

This work was financially supported by the University of Oslo, the Spanish Ministerio de Economía y Competitividad, the European FEDER Program (Projects MAT201122719 and ENE-2014-52105-R) and the Gobierno de Aragón (research group T12). The authors would like to acknowledge the use of Servicio General de Apoyo a la Investigación-SAI, Universidad de Zaragoza and to thank I. Cabistany and J. A. Gómez for technical assistance with manufacturing the tapes.

\section{References}

[1] J Nagamatsu, N Nakagawa, T Muranaka, Y Zenitani, and J Akimitsu. Superconductivity at $39 \mathrm{k}$ in magnesium diboride. Nature, 410:63, 2001.

[2] C Buzea and T Yamashita. Review of the superconducting properties of $\mathrm{MgB}_{2}$. Superconductor Science and Technology, 14(11):R115, 2001.

[3] X X Xi. $\mathrm{MgB}_{2}$ thin films. Superconductor Science and Technology, 22(4):043001, 2009.

[4] S X Dou, S Soltanian, J Horvat, X L Wang, S H Zhou, M Ionescu, H K Liu, P Munroe, and $\mathrm{M}$ Tomsic. Enhancement of the critical current density and flux pinning of $\mathrm{MgB}_{2}$ superconductor by nanoparticle $\mathrm{SiC}$ doping. Applied Physics Letters, 81(18):3419, 2002.

[5] B A Glowacki, M Majoros, M Vickers, J E Evetts, Y Shi, and I McDougall. Superconductivity of powder-in-tube $\mathrm{MgB}_{2}$ wires. Superconductor Science and Technology, 14(4):193, 2001.

[6] S Jin, H Mavoori, C Bower, and R B van Dover. High critical currents in iron-clad superconducting $\mathrm{MgB}_{2}$ wires. Nature, 411:563, 2001.

[7] H Suo, C Beneduce, M Dhallé, N Musolino, J-Y Genoud, and R Flükiger. Large transport critical currents in dense Fe- and $\mathrm{Ni}$-clad $\mathrm{MgB}_{2}$ superconducting tapes. Applied Physics Letters, 79(19):3116, 2001.

[8] R G Mints and A L Rakhmanov. Critical state stability in type-II superconductors and superconducting-normal-metal composites. Rev. Mod. Phys., 53:551-592, Jul 1981.

[9] S L Wipf. Review of stability in high temperature superconductors with emphasis on flux jumping. Cryogenics, 31(11):936 - 948, 1991.

[10] S X Dou, X L Wang, J Horvat, D Milliken, A H Li, K Konstantinov, E W Collings, M D Sumption, and H K Liu. Flux jumping and a bulk-to-granular transition in the magnetization of a compacted and sintered $\mathrm{MgB}_{2}$ superconductor. Physica C: Superconductivity, 361(2):79 83, 2001.

[11] T H Johansen, M Baziljevich, D V Shantsev, P E Goa, Y M Galperin, W N Kang, H J Kim, E M Choi, M-S Kim, and S I Lee. Dendritic magnetic instability in superconducting $\mathrm{MgB}_{2}$ films. EPL (Europhysics Letters), 59(4):599, 2002.

[12] X L Wang, S Soltanian, J Horvat, A H Liu, M J Qin, H K Liu, and S X Dou. Very fast formation of superconducting $\mathrm{MgB}_{2} / \mathrm{Fe}$ wires with high $\mathrm{J}_{c}$. Physica C: Superconductivity, 361(3):149 $155,2001$.

[13] X L Wang, Q W Yao, J Horvat, M J Qin, and S X Dou. Significant improvement of critical current density in coated $\mathrm{MgB}_{2} / \mathrm{Cu}$ short tapes through nano-SiC doping and short-time in situ reaction. Superconductor Science and Technology, 17(3):L21, 2004.

[14] C A Durán, P L Gammel, R E Miller, and D J Bishop. Observation of magnetic-field penetration via dendritic growth in superconducting niobium films. Phys. Rev. B, 52:75-78, Jul 1995. 
[15] I A Rudnev, D V Shantsev, T H Johansen, and A E Primenko. Avalanche-driven fractal flux distributions in NbN superconducting films. Applied Physics Letters, 87(4):042502, 2005.

[16] T H Johansen, M Baziljevich, D V Shantsev, P E Goa, Y M Galperin, W N Kang, H J Kim, E M Choi, M-S Kim, and S I Lee. Dendritic flux patterns in $\mathrm{MgB}_{2}$ films. Superconductor Science and Technology, 14(9):726, 2001.

[17] P Leiderer, J Boneberg, P Brüll, V Bujok, and S Herminghaus. Nucleation and growth of a flux instability in superconducting $\mathrm{YBa}_{2} \mathrm{Cu}_{3} \mathrm{O}_{7-x}$ films. Phys. Rev. Lett., 71:2646-2649, Oct 1993.

[18] U Bolz, B Biehler, D Schmidt, B-U Runge, and P Leiderer. Dynamics of the dendritic flux instability in $\mathrm{YBa}_{2} \mathrm{Cu}_{3} \mathrm{O}_{7-\delta}$ films. EPL (Europhysics Letters), 64(4):517, 2003.

[19] M R Wertheimer and J le G Gilchrist. Flux jumps in type II superconductors. Journal of Physics and Chemistry of Solids, 28(12):2509 - 2524, 1967.

[20] A J Qviller, V V Yurchenko, K Eliassen, J I Vestgården, T H Johansen, M R Nevala, I J Maasilta, $\mathrm{K}$ Senapati, and R C Budhani. Irreversibility of the threshold field for dendritic flux avalanches in superconductors. Physica C: Superconductivity, 470(19):897 - 900, 2010.

[21] C Laliena, T Qureishy, E Martínez, R Navarro, P Mikheenko, T H Johansen, and P Kováč. Effect of ball milling on the local magnetic flux distribution and microstructure of in situ $\mathrm{Fe} / \mathrm{MgB}_{2}$ conductors. Journal of Alloys and Compounds, 717:164, 2017.

[22] E Martínez, R Navarro, and J M Andrés. Improvement of the critical current density on in situ pit processed $\mathrm{Fe} / \mathrm{MgB}_{2}$ wires by oleic acid addition. Superconductor Science and Technology, 26(12):125017, 2013.

[23] Ch Jooss, J Albrecht, H Kuhn, S Leonhardt, and H Kronmüller. Magneto-optical studies of current distributions in high- $\mathrm{T}_{c}$ superconductors. Reports on Progress in Physics, 65(5):651, 2002.

[24] L E Helseth, R W Hansen, E I Il'yashenko, M Baziljevich, and T H Johansen. Faraday rotation spectra of bismuth-substituted ferrite garnet films with in-plane magnetization. Phys. Rev. B, 64:174406, Oct 2001.

[25] L E Helseth, A G Solovyev, R W Hansen, E I Il'yashenko, M Baziljevich, and T H Johansen. Faraday rotation and sensitivity of (100) bismuth-substituted ferrite garnet films. Phys. Rev. $B, 66: 064405$, Aug 2002.

[26] D V Shantsev, A V Bobyl, Y M Galperin, T H Johansen, and S I Lee. Size of flux jumps in superconducting films. Phys. Rev. B, 72:024541, Jul 2005.

[27] D V Denisov, D V Shantsev, Y M Galperin, E-M Choi, H-S Lee, S-I Lee, A V Bobyl, P E Goa, A A F Olsen, and T H Johansen. Onset of dendritic flux avalanches in superconducting films. Phys. Rev. Lett., 97:077002, Aug 2006.

[28] M S Welling, R J Westerwaal, W Lohstroh, and R J Wijngaarden. Huge compact flux avalanches in superconducting Nb thin films. Physica C: Superconductivity, 411(12):11 - 17, 2004.

[29] J I Vestgården, Y M Galperin, and T H Johansen. Oscillatory regimes of the thermomagnetic instability in superconducting films. Phys. Rev. B, 93:174511, May 2016.

[30] R G Mints and E H Brandt. Flux jumping in thin films. Phys. Rev. B, 54:12421-12426, Nov 1996.

[31] I S Aranson, A Gurevich, M S Welling, R J Wijngaarden, V K Vlasko-Vlasov, V M Vinokur, and U Welp. Dendritic flux avalanches and nonlocal electrodynamics in thin superconducting films. Phys. Rev. Lett., 94:037002, Jan 2005. 